\title{
Colour Image Blind Watermarking Scheme Based on Fast Walsh Hadamard Transform and Hessenberg Decomposition
}

\author{
Omar ABODENA*, Mary AGOYi \\ Cyprus International University, Lefkosa, KKTC, via Mersin 10, Turkey \\ omdaina@gmail.com (*Corresponding author),magoyi@ciu.edu.tr
}

\begin{abstract}
Colour image watermarking has become one of the most important algorithms for copyright protection. The following paper will present an innovative scheme for watermarking blind colour images using the discrete wavelet transform (DWT), fast Walsh Hadamard transform (FWHT) and the Hessenberg decomposition as its basis. First, two-level DWT followed by FWHT are used to decompose the host image's red channel. Next, the FWHT coefficients are split into $4 \times 4$ non-overlapping blocks. Then, each selected block is decomposed using Hessenberg decomposition, where the first row, first column element of the upper Hessenberg matrix $\mathrm{H}$ is quantified to embed the watermark information. Peak signalto-noise ratio, normalized cross-correlation and structural similarity index measure are used to evaluate the feasibility and the robustness. The experimental results have demonstrated that the proposed watermarking scheme is highly invisible with PSNR $>40 \mathrm{~dB}$, for several watermarked colour images, with a capacity of 4096 bits and execution time of $0.7415 \mathrm{~s}$. The proposed watermarking scheme is also highly resistant to both common image processing and geometrical attacks such as filtering, JPEG2000, noise adding, cropping, scaling, blurring and sharpening, and others.
\end{abstract}

Keywords: Watermarking, Wavelet transforms, Copyright protection, Matrix decomposition, Walsh Hadamard transform.

\section{Introduction}

Due to the rapid proliferation of modern communication and multimedia techniques, digital multimedia information (e.g. audio, image, video) can today be quickly and easily exchanged or distributed. As a result, digital information protection against illegal distribution, copying, and digital copyright modification has developed increasingly into a challenging and significant issue. Because of this, a variety of techniques have been presented to protect digital information rights. Digital watermarking has been considered one of the most efficient techniques for copyright protection, where the watermark information that contains the identity of the property is embedded in the digital multimedia without affecting its quality $[20 ; 33]$. Generally, digital watermarking methods can be classified according to various criteria. Taking into account their specific applications, the watermarking methods can be divided in two distinct classes: fragile and robust watermarking. Watermarking of the robust class is mainly employed for identification purposes, while fragile watermarking is usually used for the purposes of authentication[19; 25]. Similarly, depending on the techniques they use for extraction, watermarking algorithms can be classified in two further classes: blind and nonblind techniques. In non-blind techniques, the original digital multimedia information and the original watermark information are both required to obtain the extracted watermark information [26]. In blind techniques, obtaining the information for the watermark does not require either the original multimedia or watermark information [28]. In terms of the techniques they use for embedding, watermarking schemes can be further classified in two groups: spatial domain and frequency domain techniques. In spatial domain techniques [14; 34], the watermark is directly inserted by changing the intensity of original multimedia pixels. On the other hand, in frequency domain techniques, the original multimedia is converted into frequency coefficients by the use of transform domain, and the watermark is then inserted. Transform domain techniques provide greater levels of invisibility, as well as robustness against a variety of attacks in comparison with spatial domain techniques [36; 38]. Transform domain watermarking techniques have been developed using discrete wavelet transform (DWT) [11; 39], discrete Fourier transform (DFT) [5], discrete cosine transform (DCT) $[23 ; 29]$ or matrix decompositions, including singular value decomposition (SVD) [16], QR decomposition [21], LU decomposition [15], Schur decomposition [32] and Hessenberg decomposition [37]. Several watermarking schemes which use more than one transform domain technique in order to improve the performance of the watermarking method have been proposed and the combination of these transformations may compensate for each other's drawbacks [30]. Among transform domain techniques, DWT is applied to watermarking techniques more frequently than other transforms 
due to its multi-resolution characteristics and excellent spatial localisation, both of which are similar to theoretical models of the human visual system (HVS) [10; 12]. Recently, several digital watermarking schemes using a matrix decomposition have been presented in the attempt to improve robustness and invisibility. Meanwhile, QR decomposition is widely used in image watermarking techniques, as it can offer better, or at least comparable, performance than Schur decomposition or SVD [13; 35]. In order to compute QR decomposition, Hessenberg decomposition, whose computational complexity is lower than that of other types, is a necessary part of the process [31]. Another very important transform that has been used in speech and image processing is Walsh Hadamard transform (WHT) [6]. This is a highly efficient orthogonal transform, requiring only additions and subtractions, which makes it faster than sinusoidal transforms. WHT is very helpful for reducing spread-spectrum analysis and bandwidth storage requirements [17]. It also has a very efficient algorithm, fast Walsh Hadamard transform (FWHT). The FWHT offers the advantage of excellent energy compaction, increased speed of processing time and ease of implementation [24]. In the rest of this paper, a brief overview of discrete wavelet transform (DWT), fast Walsh Hadamard transform (FWHT) and Hessenberg decomposition is introduced in section 2. Next, the third section describes the extraction and embedding processes of the scheme proposed. Section 4 provides the results of experimentation using this scheme. Section 5 offers the conclusions drawn from these results.

\section{Preliminaries}

\subsection{Discrete wavelet transform}

DWT can offer a multi-resolution capacity in terms of separation of an image into a set of wavelets. The input image can be decomposed into four multi-resolution sub-bands of data, namely $\mathrm{HH}$, and into other three, corresponding to HL (horizontal detail component), LH (vertical detail component) and LL (lower resolution approximation coefficients). Each sub-band can be further decomposed into additional four subbands. The HH (diagonal detail component) subband contains the high-frequency components of the input image, while the LL sub-band contains low-frequency components of the input image.
The most contained information of the input image appears in the LL sub-band and the less contained information of the input image appears in the HH sub-band $[2 ; 8 ; 18]$.

\subsection{Fast Walsh Hadamard transform}

The Walsh Hadamard transform (WHT) is a highly efficient non-sinusoidal, orthogonal linear transform which separates a signal into a set of Walsh functions comprising rectangular or square waves with the only binary values of \pm 1 [3]. This transform has also been used as a low-complexity transform technique for image processing [27]. WHT also exists in a faster version, fast Walsh Hadamard transform (FWHT), which has $N N$ additions and subtractions for N-point input samples. FWHT is able to represent signals with sharp discontinuities more precisely, utilising less coefficients than the fast Fourier transform [9]. The Walsh Hadamard matrix $H_{N}$ of order $N$ can be defined as follows [4]:

$H_{N}=\left[\begin{array}{ll}H_{\frac{N}{2}} & \frac{H_{N}}{2} \\ \frac{H_{N}}{2} & -H_{\frac{N}{2}}\end{array}\right]$

\subsection{Hessenberg decomposition}

Hessenberg decomposition $[22 ; 31 ; 40]$ is an orthogonal matrix decomposition that has been used in certain control theory applications. Hessenberg decomposition factorises a matrix $A$ into its factors $\mathrm{Q}$ and $\mathrm{H}$, which can be presented as follows:

$A=Q H Q^{T}$

where $Q$ is an orthogonal matrix, whereas $H$ is an upper Hessenberg matrix, i.e., $h_{i, j}=0$ whenever $i>j+1$. Hessenberg decomposition has been estimated as a 'front end' factorisation for QR decomposition because the first step in computing $\mathrm{QR}$ is to compute Hessenberg decomposition. Hessenberg decomposition can be computed by Householder matrices, which are orthogonal matrices of the form

$P=\frac{\left(I_{n}-2 u u^{T}\right)}{u^{T} u}$

Here, u represents a non-zero vector in $R^{n}$ and $I_{n}$ signifies the identity matrix $n \times n$. The complete procedure comprises $n$-2 steps where $A$ is of size $n \times n$.

$$
\begin{aligned}
& H=\left(P_{1} \ldots P_{n-1}\right)^{T} A\left(P_{1} \ldots P_{n-1}\right) \\
& H=Q^{T} A Q
\end{aligned}
$$




\section{The Proposed Watermarking Scheme}

A novel scheme of blind colour image watermarking is proposed in this paper, using discrete wavelet transform (DWT), fast Walsh Hadamard transform (FWHT), and Hessenberg decomposition. Primarily, it focuses upon the process of watermark embedding and the process of watermark extraction.

\subsection{The process of watermark embedding}

Figure 1 illustrates the process of watermark embedding, using a combination of FWHT, DWT and Hessenberg decomposition, with the steps in detail being as follows:

Step 1. Divide the host colour image $H_{i}$ of size $M$ $\times M$ into $R, G$ and $B$ components, where $M=1024$.

Step 2. Decompose the $R$ component $R_{c}$ of the host image using two-level DWT as shown:

$$
\begin{aligned}
& {\left[\begin{array}{llll}
L L_{1} & L H_{1} & H L_{1} H H_{1}
\end{array}\right]=\operatorname{DWT}\left(R_{c}\right)} \\
& {\left[\begin{array}{llll}
L L_{2} & L H_{2} & H L_{2} & H H_{2}
\end{array}\right]=D W T\left(H H_{1}\right)}
\end{aligned}
$$

Step 3. Apply FWHT to high-frequency sub-band $\mathrm{HH}_{2}$ to obtain the FWHT coefficients.

Step 4. Divide the FWHT coefficients into $4 \times 4$ non-overlapping blocks. Total number of blocks is $64^{2}$.
Step 5. Convert the $N \times N$ watermark image into a binary watermark of size $N \times N$, where $N=64$.

Step 6. Apply Hessenberg decomposition to each selected $4 \times 4$ block according to Eq. (2) to obtain the matrices $Q$ and $H$.

Step 7. Modify magnitudes $M_{1}$ and $M_{2}$ according to the watermark information $w$, as follows:

if $w(i, j)=1,\left\{\begin{array}{l}M_{1}=0.5 \alpha \\ M_{2}=-1.5 \alpha\end{array}\right.$
if $w(i, j)=0,\left\{\begin{array}{l}M_{1}=-0.5 \alpha \\ M_{2}=1.5 \alpha\end{array}\right.$

where $(1 \leq i, j \leq N)$ and $\alpha$ denotes the quantisation step.

Step 8. The possible quantisation results $T_{l}$ and $T_{2}$ can be computed by the modifying magnitudes $M_{1}$ and $M_{2}$.

$T_{1}=2 k \alpha+M_{1}$

$T_{2}=2 k \alpha+M_{2}$

where $k=$ floor $\left(\operatorname{ceil}\left(h_{11} / \alpha\right) / 2\right), \operatorname{ceil}($. obtains the largest nearest integer, and floor(.) obtains the least nearest integer.

Step 9. Select the first row, first column element $h_{1,1}$ of matrix $H$ to embed the binary watermark as shown:

$$
\begin{aligned}
& h_{1,1}^{\prime}= \\
& \left\{\begin{array}{l}
T_{2} \text { if abs }\left(h_{1,1}-T_{2}\right)<\operatorname{abs}\left(h_{1,1}-T_{1}\right) \\
T_{1} \text { else }
\end{array}\right.
\end{aligned}
$$

where abs(.) denotes the absolute value.

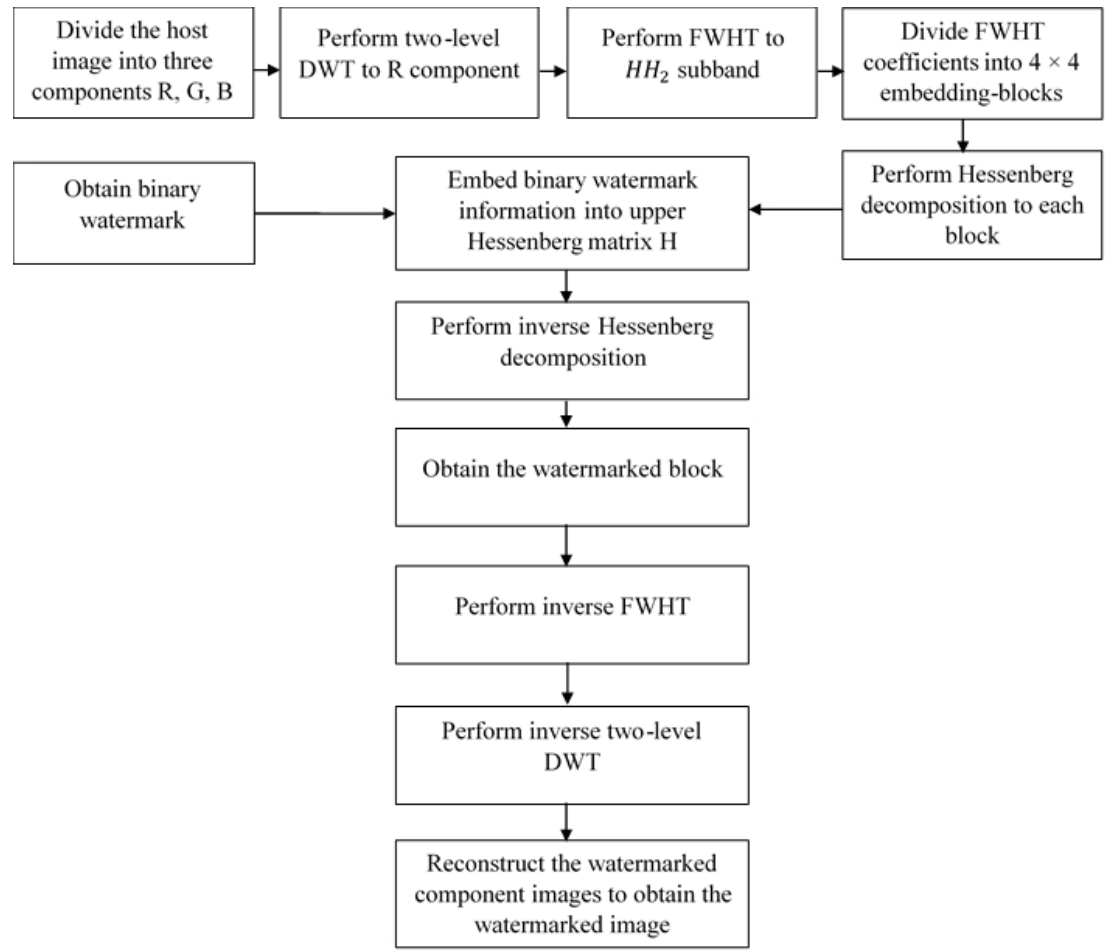

Fig. 1. Block diagram of the embedding process 
Step 10. Replace $h_{1,1}$ with $h_{1,1}^{\prime}$ and then apply the inverse of Hessenberg decomposition to get the watermarked block $A$ '.

$A^{\prime}=Q H^{\prime} Q^{T}$

Step 11. Repeat the embedding steps 6-10 until all watermark information is embedded.

Step 12. Combine all watermarked blocks together.

Step 13. Apply the inverse of FWHT to the combined watermarked blocks to obtain the $H H^{\mathrm{w}}$ sub-band, where $H H^{\mathrm{w}}$ denotes the watermarked approximate sub-band.

Step 14. Apply inverse two-level DWT using the modified high-frequency sub-band $\mathrm{HH}^{\mathrm{w}}$ instead of $\mathrm{HH}_{2}$ as shown:

$$
\begin{aligned}
& X=I D W T\left[L L_{2} L H_{2} H L_{2} H H^{w}\right] \\
& R_{c}^{\prime}=I D W T\left[L L_{1} L H_{1} H L_{1} X\right]
\end{aligned}
$$

Step 15. Reconstruct the watermarked $R, G$ and $B$ channels to have the watermarked colour image $O^{\prime}$.

\subsection{The process of watermark extraction}

Figure 2 illustrates the process for watermark extraction, with the steps in detail being as follows:

Step 1. Divide the watermarked colour image $O$ into $R, G$ and $B$ components.

Step 2. Decompose the $R$ component $R_{c}{ }_{c}$ of the watermarked colour image using twolevel DWT, as follows:

$$
\begin{aligned}
& {\left[\begin{array}{llll}
L L_{1} & L H_{1} H L_{1} H H_{1}
\end{array}\right]=\operatorname{DWT}\left(R^{\prime \prime}{ }_{c}\right)} \\
& {\left[\begin{array}{lll}
L L_{2} & L H_{2} & H L_{2} H H_{2}
\end{array}\right]=\operatorname{DWT}\left(H H_{1}\right)}
\end{aligned}
$$

Step 3. Apply FWHT to high-frequency sub-band $\mathrm{HH}_{2}$ to obtain the FWHT coefficients.

Step 4. Divide the FWHT coefficients into $4 \times 4$ non-overlapping blocks.

Step 5. Apply Hessenberg decomposition to each selected $4 \times 4$ block according to Eq. (2) to obtain the matrices $Q^{\prime}$ and $H^{\prime}$.

Step 6. Use the first row, first column element $h_{1,1}^{\prime}$ of $H^{\prime}$ matrix to extract the binary watermark as shown:

$w_{(i, j)}^{\prime}=\bmod \left(\operatorname{ceil}\left(\frac{h^{\prime}{ }_{1,1}}{\alpha}\right), 2\right)$

where $(1 \leq i, j \leq N)$ and $\bmod ($.) denotes the modulo operation.

Step 7. Repeat the extracting steps 5-6 until all watermark information is extracted. The extracted binary watermark is converted to the extracted watermark image.

\section{Experimental Results}

In this section, the performances of the proposed blind colour image watermarking scheme are analysed by measuring their feasibility and robustness. All results were derived from experiments conducted on a laptop computer which had $2.00 \mathrm{GHz}$ CPU Intel Core i3 with 4.00 GB RAM, using MATLAB R2017b in Windows 10 environment. All host images are 24-bit $1024 \times 1024$ colour images in the USCSIPI image database [41] such as Lena, Baboon, Peppers, Sailboat, and Airplane, as shown in Figures 3(a-e), respectively. An image of size $64 \times 64$ is utilised as watermark, as shown in Figure 3(f). This image was used in [34; 39]. To

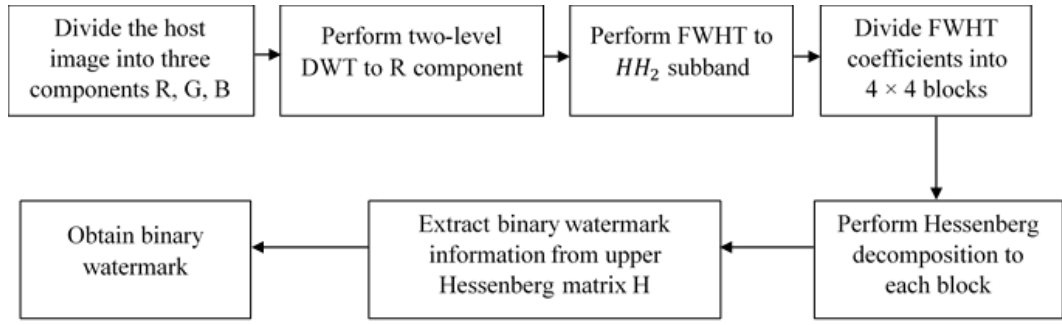

Fig. 2. Block diagram of the extraction process

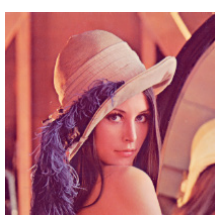

Lena

a

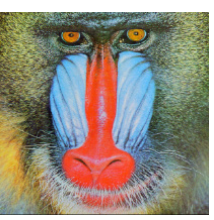

Baboon

$b$

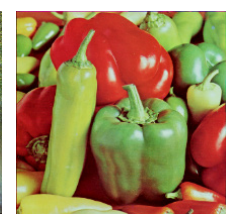

Peppers

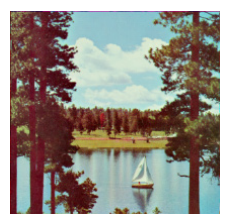

Sailboat

$d$

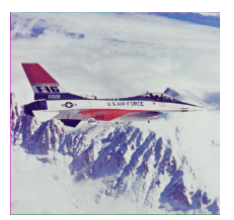

Airplane

$e$

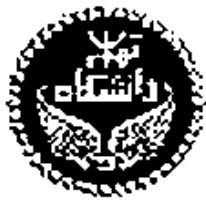

Watermark

$f$

Fig. 3. The host colour images and the watermark image 

Watermarked
image

Extracted watermark
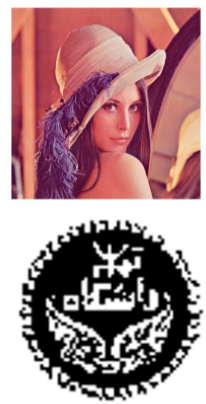
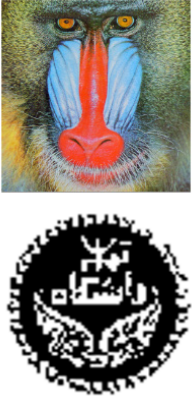
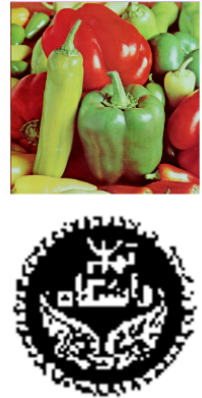
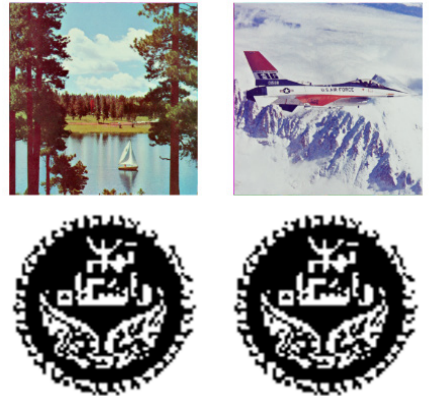

Fig. 4. Embedding and extraction of watermark without any attacks

measure the feasibility and robustness capabilities of the proposed colour image watermarking scheme, the peak signal-to-noise ratio (PSNR) [1] and the structural similarity index measure (SSIM) [7] are used to evaluate the invisibility of the reconstructed watermarked colour image. Meanwhile, normalised cross-correlation (NC) [21] is utilised to evaluate the similarity between the corresponding final watermark image and the original watermark image. The performance metrics are mathematically defined as follows.

$P S N R=10 \log _{10} \frac{S^{2}}{M S E}$

where $S$ is the peak signal value, MSE is the mean square error given by

$M S E=\frac{1}{M N} \sum_{x=1}^{M} \sum_{y=1}^{N}\left[H(x, y)-H^{\prime}(x, y)\right]^{2}$

$H(x, y)$ and $H^{\prime}(x, y)$ represents the intensity value of pixel at location $(M, N)$ in the host image and the watermarked image, respectively. The PSNR value for colour images can be computed as follows.

$P S N R_{R G B}=\frac{1}{3}\left(P S N R_{R}+P S N R_{G}+P S N R_{B}\right)$

where $\mathrm{PSNR}_{\mathrm{R}}, \mathrm{PSNR}_{\mathrm{G}}$ and $\mathrm{PSNR}_{\mathrm{B}}$ are PSNR value for $R, G$ and $B$ componets, respectively.

$\operatorname{SSIM}(X, Y)=\frac{1}{3} \sum_{\xi \in\{R, G, B\}} \operatorname{SSIM}\left(X_{\xi}, Y_{\xi}\right)$

where $X$ and $Y$ are host colour image and watermarked colour image, respectively.

$\operatorname{SSIM}\left(X_{\xi}, Y_{\xi}\right)=\frac{\left(\mu_{X_{\xi}} \mu_{Y_{\xi}}+C_{1}\right)\left(2 \sigma_{X_{\xi} Y}+C_{2}\right)}{\left(\mu_{X_{\xi}}^{2}+\mu_{Y_{\xi}}^{2}+C_{1}\right)\left(\sigma_{X_{\xi}}^{2}+\sigma_{Y}^{2}+C_{2}\right)}$

where $\mu X_{\xi}$ and $\mu Y_{\xi}$ are the average of $X_{\xi}$ and $Y_{\xi}$ respectively. $\sigma X_{\xi}$ and $\sigma Y_{\xi}$ are the variance of $X_{\xi}$ and $Y_{\xi}$ respectively. $\sigma X_{\xi} Y_{\xi}$ is the covariance of $X_{\xi}^{\xi}$ and $Y_{\xi}^{\zeta}$ and $C_{1}$ and $C_{2}$ are two variables utilised to avoid the denominator being zero.

$$
N C=\frac{\sum_{i=1}^{m} \sum_{j=1}^{n} w(i, j) \times w^{\prime}(i, j)}{\sqrt{\sum_{i=1}^{m} \sum_{j=1}^{n}[w(i, j)]^{2}} \times \sqrt{\sum_{i=1}^{m} \sum_{j=1}^{n}\left[w^{\prime}(i, j)\right]^{2}}}
$$

Here, $w$ and $w^{\prime}$ are the original watermark image and extracted one, respectively.

Table 1 The PSNR, SSIM, and NC values without any attacks

\begin{tabular}{|l|l|l|l|}
\hline \multicolumn{1}{|c|}{ Test image } & \multicolumn{1}{c|}{ PSNR } & \multicolumn{1}{c|}{ SSIM } & \multicolumn{1}{c|}{ NC } \\
\hline Lena & 40.159 & 1.000 & 1.000 \\
\hline Baboon & 40.130 & 1.000 & 1.000 \\
\hline Peppers & 40.156 & 1.000 & 1.000 \\
\hline Sailboat & 40.153 & 1.000 & 1.000 \\
\hline Airplane & 40.159 & 1.000 & 1.000 \\
\hline
\end{tabular}

Generally, a higher SSIM or PSNR value denotes that the proposed digital watermarking scheme has more feasibility. In addition, a higher NC value denotes that the proposed digital watermarking scheme has higher robustness. The main objective function of the proposed scheme is to focus on both invisibility and robustness. Because of this, the watermark is embedded in the red channel instead of green or blue channel to obtain the best result in both invisibility and robustness. As such, the watermark quantisation step $\alpha=0.034$ is used to obtain higher values of PSNR, SSIM and NC. The image qualities (SSIM and PSNR values) of the corresponding watermarked colour images without any attacks are listed in Table 1. Meanwhile, the NC values of the final watermarks that are extracted from the corresponding watermarked colour images without any attacks are also listed to prove the effectiveness of the proposed watermarking scheme. The corresponding watermarked colour images without any attacks, and the retrieved watermarks, are shown in Figure 4. As can be seen from Table 1 and Figure 4, the corresponding watermarked colour images have better feasibility 
Table 2 The SSIM values of watermarked colour images under common attacks.

\begin{tabular}{|l|l|l|l|l|l|}
\hline \multicolumn{1}{|c|}{ Attack } & \multicolumn{1}{|c|}{ Lena } & Baboon & Peppers & Sailboat & Airplane \\
\hline Flipping up to down & 0.993 & 0.991 & 0.985 & 0.984 & 0.994 \\
\hline Sharpening (1) & 1.000 & 1.000 & 1.000 & 1.000 & 1.000 \\
\hline Blurring (1) & 1.000 & 1.000 & 1.000 & 1.000 & 1.000 \\
\hline Salt \& Pepper noise (0.01) & 1.000 & 1.000 & 1.000 & 1.000 & 1.000 \\
\hline Gamma correction (2.0) & 0.996 & 0.995 & 0.996 & 0.996 & 0.997 \\
\hline Gaussian noise [0,0.01] & 1.000 & 1.000 & 1.000 & 1.000 & 1.000 \\
\hline Poisson noise & 1.000 & 1.000 & 1.000 & 1.000 & 1.000 \\
\hline Median filter (3×1) & 1.000 & 1.000 & 1.000 & 1.000 & 1.000 \\
\hline Scaling (7) & 1.000 & 1.000 & 1.000 & 1.000 & 1.000 \\
\hline Cropping (80\%) & 0.964 & 0.968 & 0.970 & 0.966 & 0.940 \\
\hline JPEG2000 (CompressionRatio=5) & 1.000 & 1.000 & 1.000 & 1.000 & 1.000 \\
\hline
\end{tabular}

Table 3 The NC values of the final watermarks when subject to common attacks.

\begin{tabular}{|l|l|l|l|l|l|}
\hline \multicolumn{1}{|c|}{ Attack } & Lena & Baboon & Peppers & Sailboat & Airplane \\
\hline Flipping up to down & 1.000 & 1.000 & 1.000 & 1.000 & 1.000 \\
\hline Sharpening (1) & 1.000 & 1.000 & 1.000 & 1.000 & 1.000 \\
\hline Blurring (1) & 1.000 & 0.986 & 1.000 & 1.000 & 1.000 \\
\hline Salt \& Pepper noise (0.01) & 1.000 & 1.000 & 1.000 & 1.000 & 1.000 \\
\hline Gamma correction (2.0) & 1.000 & 1.000 & 1.000 & 1.000 & 1.000 \\
\hline Gaussian noise [0,0.01] & 0.990 & 0.988 & 0.991 & 0.984 & 0.987 \\
\hline Poisson noise & 1.000 & 1.000 & 1.000 & 1.000 & 1.000 \\
\hline Median filter (3×1) & 0.995 & 0.996 & 0.989 & 0.992 & 0.995 \\
\hline Scaling (7) & 1.000 & 1.000 & 1.000 & 1.000 & 1.000 \\
\hline Cropping (80\%) & 1.000 & 1.000 & 1.000 & 1.000 & 1.000 \\
\hline JPEG2000 (Compression Ratio=5) & 1.000 & 0.999 & 1.000 & 0.999 & 1.000 \\
\hline
\end{tabular}

Table 4 Invisibility and robustness of the scheme proposed compared to the existing methods [34; 39]

\begin{tabular}{|c|l|l|l|l|l|l|c|c|}
\hline \multirow{3}{*}{ Image } & \multirow{2}{*}{ Algorithm } & \multirow{2}{*}{ PSNR } & \multirow{2}{*}{ SSIM } & \multicolumn{6}{|c|}{ NC } \\
\cline { 5 - 10 } & & & & $\begin{array}{c}\text { Gaussian } \\
\text { noise }\end{array}$ & $\begin{array}{c}\text { Poisson } \\
\text { noise }\end{array}$ & $\begin{array}{c}\text { Median } \\
\mathbf{3} \times \mathbf{3}\end{array}$ & $\begin{array}{c}\text { Crop } \\
\mathbf{1 0 \%}\end{array}$ & $\begin{array}{c}\text { Scale } \\
\times \mathbf{0 . 8}\end{array}$ \\
\hline \multirow{4}{*}{ Lena } & {$[39]$} & 41.742 & NA & 0.920 & 0.956 & 0.978 & 0.937 & 0.947 \\
\cline { 3 - 10 } & {$[34]$} & NA & 0.986 & 0.967 & NA & 0.967 & 0.987 & 0.994 \\
\cline { 2 - 10 } & Proposed & 40.159 & 1.000 & 0.990 & 1.000 & 0.983 & 1.000 & 1.000 \\
\hline \multirow{4}{*}{ Baboon } & {$[39]$} & 39.312 & NA & 0.851 & 0.938 & 0.880 & 0.924 & 0.860 \\
\cline { 2 - 10 } & {$[34]$} & NA & 0.995 & 0.963 & NA & 0.996 & 0.983 & 0.944 \\
\cline { 2 - 10 } & Proposed & 40.130 & 1.000 & 0.988 & 1.000 & 0.945 & 1.000 & 1.000 \\
\hline \multirow{3}{*}{ Peppers } & {$[39]$} & 39.272 & NA & 0.919 & 0.949 & 0.959 & 0.928 & 0.959 \\
\cline { 2 - 10 } & {$[34]$} & NA & 0.985 & 0.954 & NA & 0.968 & 0.979 & 0.982 \\
\cline { 2 - 10 } & Proposed & 40.156 & 1.000 & 0.991 & 1.000 & 0.962 & 1.000 & 1.000 \\
\hline
\end{tabular}

and are similar to the host colour images under visual observation. As it can also be observed from Figure 4, the watermark information can be effectively embedded into the host colour images and the hidded watermark information can be almost fully extracted from the corresponding watermarked colour images. To investigate the robustness of the proposed watermarking scheme, both geometrical distortions and image processing operations have been performed on the corresponding watermarked colour images. These include sharpening, flipping, blurring, Gamma 
correction, salt and pepper noise, Poisson noise, Gaussian noise, scaling, cropping, median filter and JPEG2000 compression attacks. Table 2 lists the SSIM results of attacked watermarked colour images. As can be seen from Table 2, the proposed watermarking scheme is fairly imperceptible when attacked. Meanwhile, the NC results of the final watermark from the corresponding watermarked colour images when subject to various attacks are listed in Table 3. As can be seen from Table 3, the proposed watermarking scheme demonstrates a superior capacity to resist both image processing and geometrical attacks. It can be concluded from the experimental results from Tables 1 and 2 that the proposed watermarking scheme has a higher feasibility and a stronger robustness resistance to attacks. Figures 5 to 15 show the corresponding watermarked colour images under various attacks and the watermarks that are extracted from attacked watermarked colour images under visual observation. As it can be determined from these Figures, attacked watermarked colour images are most similar to the host colour images under all attacks except the cropping attacks, and the extracted watermarks have higher similarity to the original watermark, which can be easily recognised by the human eye. To investigate the extent of the improvement offered by the proposed scheme in terms of imperceptibility and robustness, our scheme is compared with existing methods [34; 39], and the performance comparison is presented in Table 4. It is obvious that the proposed scheme outperforms the scheme proposed in $[34 ; 39]$. The capacity of the proposed colour image watermarking scheme is analysed by computing the embedding rate in bits per pixel (bpp). Since an image of size $64 \times 64$ is utilised as watermark and $1024 \times 1024$ colour images are used as host images in this experiment, the capacity is $(64 \times$ $64) /(1024 \times 1024 \times 3)=0.0013(\mathrm{bpp})$. It can be readily observed that the proposed scheme has low capacity, which is because $4 \times 4$ non-overlapping blocks are used in this scheme and one block contains only 1-bit watermark information. The execution time of the proposed colour image watermarking scheme is also analysed by computing the extraction and embedding times in seconds. Since the proposed watermarking scheme uses two-level discrete wavelet transform, which needs more execution time for performing inverse-transformation, the time is somewhat extended. However, the proposed scheme has the embedding and extraction times of $0.5355 \mathrm{~s}$ and $0.2060 \mathrm{~s}$, respectively. We can see, then, that the proposed scheme still has a low execution time, which happens because it uses FWHT and Hessenberg decomposition. These offer short execution times, and are able to compensate for the time-related drawbacks of two-level DWT.

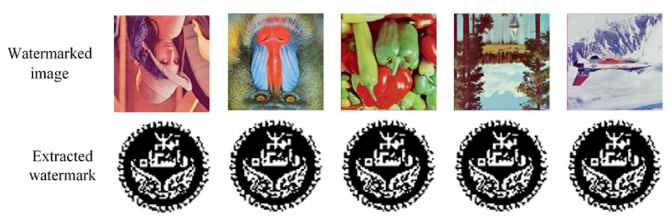

Fig. 5. Embedding and extraction of watermark under flipping attack

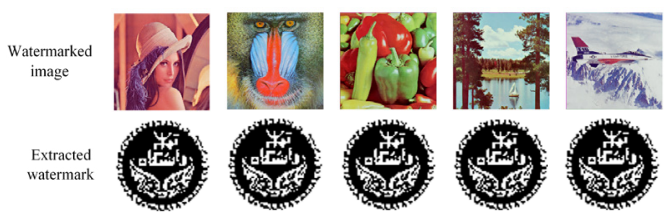

Fig. 6. Embedding and extraction of watermark under sharpening attack

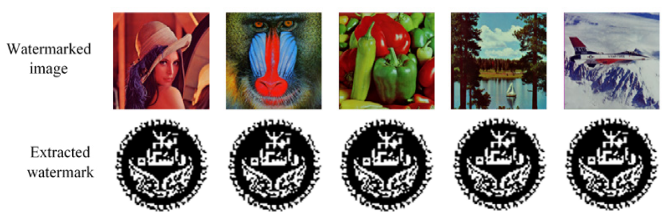

Fig. 7. Embedding and extraction of watermark under Gamma correction attack

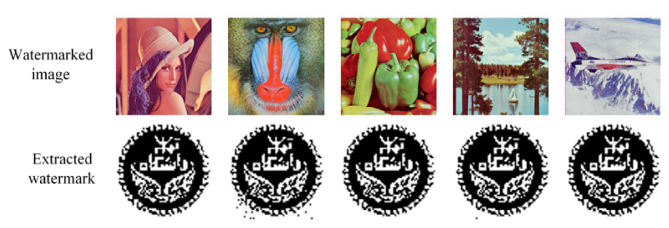

Fig. 8. Embedding and extraction of watermark under blurring attack

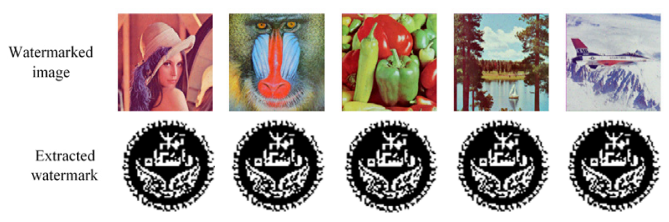

Fig. 9. Embedding and extraction of watermark under salt \& pepper noise attack

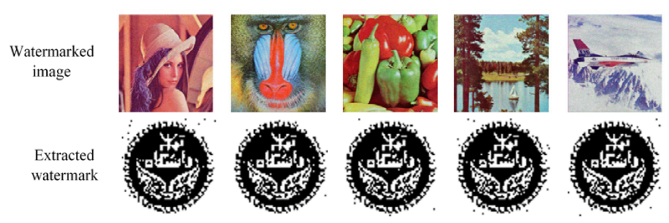

Fig. 10. Embedding and extraction of watermark under Gaussian noise attack 


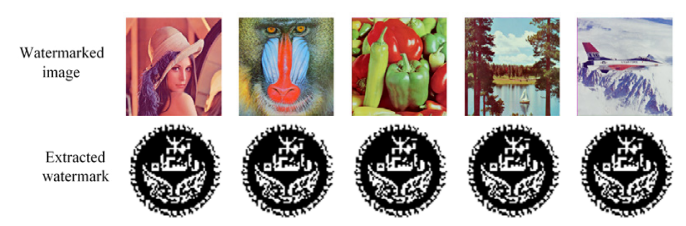

Fig. 11. Embedding and extraction of watermark under Poisson noise attack

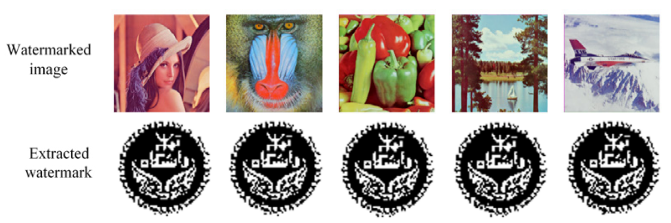

Fig. 12. Embedding and extraction of watermark under median filter attack

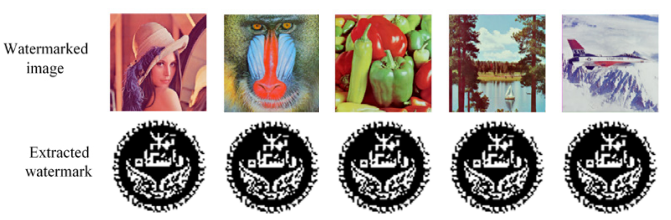

Fig. 13. Embedding and extraction of watermark under scaling attack

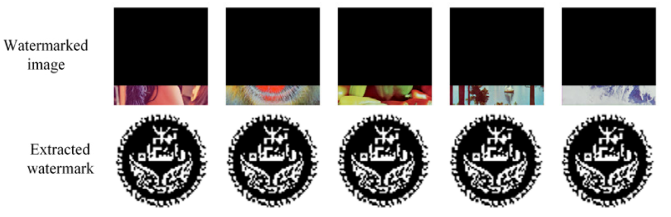

Fig. 14. Embedding and extraction of watermark under cropping attack

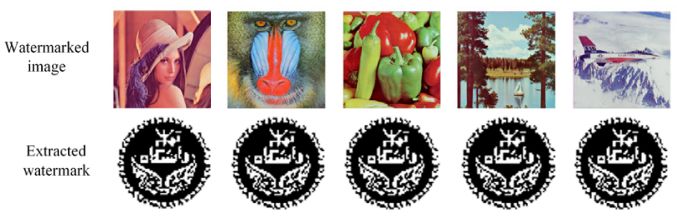

Fig. 15. Embedding and extraction of watermark under JPEG2000 compression attack

\section{REFERENCES}

1. Al-Otum, H. M. \& Samara, N. A. (2010). A robust blind color image watermarking based on wavelet-tree bit host difference selection, Signal Processing, 90(8), 2498-2512.

2. Ali, M. \& Ahn, C. W. (2014). An optimized watermarking technique based on selfadaptive DE in DWT-SVD transform domain, Signal Processing, 94, 545-556.

3. Bin, Y., Xingming, S., Xianyi, C., Zhang, J. \& Xu, L. (2013). An Efficient Forensic Method for Copy--move Forgery Detection Based on DWT-FWHT, Radioengineering, 22(4).

\section{Conclusion}

This paper has described a blind colour image watermarking scheme utilising DWT in combination with the FWHT and Hessenberg decomposition, intending to deliver robust copyright protection. When applying the embedding technique, the red channel of the 24bit colour host images are decomposed by twolevel DWT and a high-frequency sub-band of the second-level DWT is transformed by FWHT. Then, FWHT coefficients are divided into 4 $\times 4$ non-overlapping blocks and each block is decomposed by Hessenberg decomposition, where the watermark information is embedded in the first row, first column element of the upper Hessenberg matrix $H$. The advantages of this method are: (1) the proposed watermarking scheme is simpler and more efficient in invisibility, robustness and computational complexity. (2) Without requiring either the host colour image or the watermark image, the watermark can be extracted successfully from watermarked colour images after subjecting them to various attacks. What is indicated by the experimental data is that the scheme which has been proposed will attain higher performances in the areas of robustness and invisibility when compared with the existing methods. In a future work, the means of embedding the watermark colour image into the colour image will be considered.

4. Boules, R. (1989). Adaptive filtering using the fast Walsh-Hadamard transformation, IEEE transactions on electromagnetic compatibility, 31(2), 125-128.

5. Cedillo-Hernández, M., García-Ugalde, F., Nakano-Miyatake, M. \& Pérez-Meana, H. M. (2014). Robust hybrid color image watermarking method based on DFT domain and 2D histogram modification, Signal, Image and Video Processing, 8(1), 49-63.

6. Chang, L.-W. \& Wu, M.-C. (1993). A bit level systolic array for Walsh-Hadamard transforms, Signal Processing, 31(3), 341-347. 
7. Chen, B., Zhou, C., Jeon, B., Zheng, Y. \& Wang, J. (2017). Quaternion discrete fractional random transform for color image adaptive watermarking, Multimedia Tools and Applications, 1-29.

8. Chiorean, L. D., Suta, L., Vaida, M. F. \& Hedesiu, M. (2010). Medical Fusion Components for a Web Dedicated Application, Studies in Informatics and Control, 19(4), 435-444.

9. Deepa, T. \& Kumar, R. (2014). Application of the joint fast Walsh Hadamard-inverse fast Fourier transform in a LDPC coded OFDM wireless communication system: Performance and complexity analysis, Wireless personal communications, 77(4), 3177-3192.

10. Dejey, D. \& Rajesh, R. (2011). Robust discrete wavelet-fan beam transforms-based colour image watermarking, IET Image Processing, 5(4), 315-322.

11. Giri, K. J. \& Bashir, R. (2017). A block based watermarking approach for color images using discrete wavelet transformation, International Journal of Information Technology, 1-8.

12. Guo, Y. \& Li, B.-Z. (2016). Blind image watermarking method based on linear canonical wavelet transform and $\mathrm{QR}$ decomposition, IET image processing, 10(10), 773-786.

13. Guo, Y., Li, B.-Z. \& Goel, N. (2017). Optimised blind image watermarking method based on firefly algorithm in DWT-QR transform domain, IET Image Processing.

14. Hua, C.-H., Tu, N. A., Hur, T., Bang, J., Kim, D., Amin, M. B., Kang, B. H., Seung, H., \& Lee, S. (2018). Selective bit embedding scheme for robust blind color image watermarking, Information Sciences, 426, $1-18$.

15. Jane, O. \& Elbaşi, E. (2014). A new approach of nonblind watermarking methods based on DWT and SVD via LU decomposition, Turkish Journal of Electrical Engineering \& Computer Sciences, 22(5), 1354-1366.

16. Jia, S.-1. (2014). A novel blind color images watermarking based on SVD, OptikInternational Journal for Light and Electron Optics, 125(12), 2868-2874.
17. Kekre, H., Kulkarni, V., Venkatraman, S., Priya, A. \& Narashiman, S. (2011). Speaker identification using row mean of DCT and walsh hadamard transform, International Journal on Computer Science and Engineering, 3(1).

18. Khalili, M. \& Asatryan, D. (2013). Colour spaces effects on improved discrete wavelet transform-based digital image watermarking using Arnold transform map, IET Signal Processing, 7(3), 177-187.

19. Lakrissi, Y., Saaidi, A. \& Essahlaoui, A. (2017). Novel dynamic color image watermarking based on DWT-SVD and the human visual system, Multimedia Tools and Applications, 1-25.

20. Li, J., Yu, C., Gupta, B. \& Ren, X. (2017). Color image watermarking scheme based on quaternion Hadamard transform and Schur decomposition, Multimedia Tools and Applications, 1-17.

21. Mehta, R., Rajpal, N. \& Vishwakarma, V. P. (2016). LWT-QR decomposition based robust and efficient image watermarking scheme using Lagrangian SVR, Multimedia Tools and Applications, 75(7), 4129-4150.

22. Moghimi, M. K. \& Pourghassem, H. (2015). Shadow Detection Based on Combinations of Hessenberg Decomposition and Principal Component Analysis in Surveillance Applications, IETE Journal of Research, 61(3), 269-284.

23. Moosazadeh, M. \& Ekbatanifard, G. (2017). An improved robust image watermarking method using DCT and YCoCg-R color space, Optik-International Journal for Light and Electron Optics, 140, 975-988.

24. Niveditha, G., Sharmila, B., Manikantan, K. \& Ramachandran, S. (2015). DWT-based face recognition using fast walsh hadamard transform and chiral image superimposition as pre-processing techniques. In $2^{\text {nd }}$ International Conference on Electronics and Communication Systems (ICECS), 2015 (pp. 7-14). IEEE.

25. Pan-Pan, N., Xiang-Yang, W., Yu-Nan, L. \& Hong-Ying, Y. (2017). A robust color image watermarking using local invariant significant bitplane histogram, Multimedia Tools and Applications, 76(3), 3403-3433. 
26. Patvardhan, C., Kumar, P. \& Lakshmi, C. V. (2017). Effective Color image watermarking scheme using $\mathrm{YCbCr}$ color space and $\mathrm{QR}$ code, Multimedia Tools and Applications, $1-23$.

27. Pauchard, Y., Cintra, R. J., Madanayake, A. \& Bayer, F. M. (2015). Fast computation of residual complexity image similarity metric using low-complexity transforms, IET Image Processing, 9(8), 699-708.

28. Rawat, S. \& Raman, B. (2012). A blind watermarking algorithm based on fractional Fourier transform and visual cryptography, Signal Processing, 92(6), 1480-1491.

29. Roy, S. \& Pal, A. K. (2017). A blind DCT based color watermarking algorithm for embedding multiple watermarks, $A E U$ International Journal of Electronics and Communications, 72, 149-161.

30. Roy, S. \& Pal, A. K. (2018). An SVD Based Location Specific Robust Color Image Watermarking Scheme Using RDWT and Arnold Scrambling, Wireless Personal Communications, 98(2), 2223-2250.

31. Su, Q. (2016). Novel blind colour image watermarking technique using Hessenberg decomposition, IET Image Processing, 10(11), 817-829.

32. Su, Q. \& Chen, B. (2017). An improved color image watermarking scheme based on Schur decomposition, Multimedia Tools and Applications, 76(22), 24221-24249.

33. Su, Q. \& Chen, B. (2018). Robust color image watermarking technique in the spatial domain, Soft Computing, 22(1), 91-106.
34. Su, Q., Niu, Y., Wang, Q. \& Sheng, G. (2013). A blind color image watermarking based on DC component in the spatial domain, OptikInternational Journal for Light and Electron Optics, 124(23), 6255-6260.

35. Su, Q., Niu, Y., Wang, G., Jia, S. \& Yue, J. (2014). Color image blind watermarking scheme based on QR decomposition, Signal Processing, 94, 219-235.

36. Su, Q., Wang, G., Zhang, X., Lv, G. \& Chen, B. (2017). A new algorithm of blind color image watermarking based on LU decomposition, Multidimensional Systems and Signal Processing, 1-20.

37. Su, Q., Wang, G., Lv, G., Zhang, X., Deng, G. \& Chen, B. (2017). A novel blind color image watermarking based on Contourlet transform and Hessenberg decomposition, Multimedia Tools and Applications, 76(6), 8781-8801.

38. Thakkar, F. N. \& Srivastava, V. K. (2017). A blind medical image watermarking: DWTSVD based robust and secure approach for telemedicine applications, Multimedia Tools and Applications, 76(3), 3669-3697.

39. Vahedi, E., Zoroofi, R. A. \& Shiva, M. (2012). Toward a new wavelet-based watermarking approach for color images using bio-inspired optimization principles, Digital Signal Processing, 22(1), 153-162.

40. Van Loan, C. (1982). Using the Hessenberg decomposition in control theory. In Algorithms and Theory in Filtering and Control (pp. 102-111). Springer.

41. The USC-SIPI image database. <http://sipi. usc.edu/database/>, accessed February 2018. 